\title{
Application of an Electromagnetic Modeling Method for Railway Grounding Systems Subjected to Lightning Strikes
}

\author{
Pieter Jacqmaer, Christophe Geuzaine and Johan Driesen, Member, IEEE
}

\begin{abstract}
This document presents the work done for the Belgian railway operator Infrabel with respect to the modeling of grounding systems. Infrabel needed information on how to design the lightning protection systems of the recently deployed GSM-R installations. There exist many methods for simulating lightning strikes on grounding systems. One of these techniques, an electromagnetic method based on Moment Methods, is used to solve the problem. The theory behind the method is described and a novel technique is presented for simulating cylindrical cables without metal sheets. The method is applied to the grounding of the Infrabel GSM-R base station. The influence of the soil conductivity, the length of cables and grounding rods and the type of lightning strike on the effectiveness of the grounding system is researched.
\end{abstract}

Index Terms-Grounding, grounding electrodes, lightning, moment methods, power system lightning effects

\section{FORMULATION OF THE PROBLEM}

I NFRABEL, the infrastructure company which manages the Belgian railway lines, recently installed approximately 480 GSM-R base-transceiver stations (BTS) across Belgium. The purpose of this cellular network is to have a communication system for the personnel and a system for the transmission of signaling information (detection of trains and controlling switches). The nominal power of the BTS is $1.3 \mathrm{kVA}$ and it is fed by a single-phase grid of $230 \mathrm{~V}$. This voltage is rectified and stepped down in order to feed the BTS at $48 \mathrm{~V}$. There are several options for supplying the BTS with electrical power, the most important one being a dedicated local connection with the distribution system. However, in some cases, because of economical reasons, another technique is used where the BTS is fed from a supply station that was normally used for the electrical supply of critical signaling infrastructure (SI). This latter situation is presented in Fig. 1. There is a cable between the supply station and the BTS and the SI is supplied from the same bus as the BTS. The supply station is a

This work was part of a project supported by the Interuniversitary Attraction Poles (IAP) programme and Infrabel N.V.

Pieter Jacqmaer and Johan Driesen are with K.U.Leuven, Department of Electrical Engineering, research group ELECTA, Kasteelpark Arenberg 10, 3001 Heverlee, Belgium (e-mail: pieter.jacqmaer@esat.kuleuven.be and johan.driesen@esat.kuleuven.be).

Christophe Geuzaine is with University of Liège, Dept. of Electrical Engineering and Computer Science (Montefiore Institute), Sart-Tilman Bldg. B28 Parking P32, B-4000 Liège, Belgium (e-mail: cgeuzaine@ulg.ac.be ). redundant three-phase IT-system at $1000 \mathrm{~V}$. Normally, the SI is well protected against overcurrents and -voltages due to lightning because of the type of grounding and because the supply station is situated in a zone of overhead wires, connected with each other through an overhead grounding wire. The BTS have antennas on metal towers and are therefore prone to lightning strikes. Therefore, if the supply station also feeds the BTS, the chances of overvoltages in the station increase.

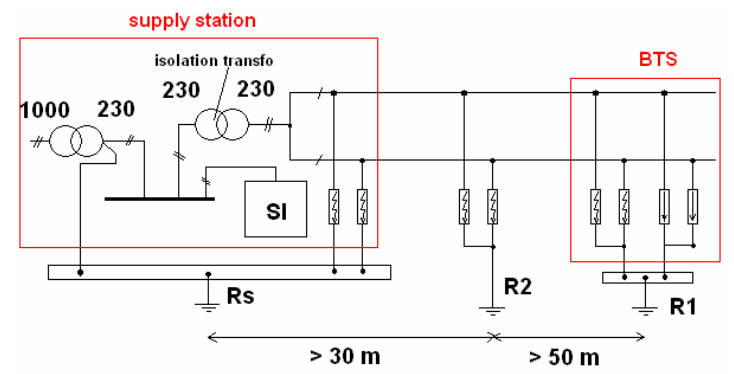

Fig. 1. Topology of the supply and grounding system of the signaling infrastructure and the GSM base station.

In order to provide a good protection against lighting, the BTS and the supply station are kept away from each other as well as possible. Infrabel enforces a minimum distance between BTS and supply station of 80 meters. Current arrestors are installed at both stations and voltage arrestors at the supply post. An isolation transformer is also chosen and installed in the supply post. The site of the BTS is grounded with a $20 \mathrm{~m}^{2}$ loop to which typically 4 grounding rods are connected. However, the design of the lightning protection system is based on common-sense rules and it is not clear whether the protection system was in fact effective. Thus, it is very important that grounding systems can be modeled accurately and when excited by lightning strikes, its behavior can be well predicted.

\section{Modeling GROUNDING Systems}

\section{A. Literature overview}

For the modeling of grounding systems, there basically exist three families of methods. First, there are methods based on circuit theory where grounding electrodes or rods are modeled with lumped or distributed components ([1]-[3]). These methods propose an easy model for grounding systems, are fast and work directly in the time-domain. Furthermore, they 
allow a good modeling of soil ionization and nonlinearities such as in the behavior of surge arrestors. However, the accuracy of these methods is rather doubtful, because the uniform model of a filter network or transmission line is used in order to describe a grounding electrode which is inherently non-uniform. Furthermore, it is difficult to describe grounding structures other than straight conductors with this technique.

Second, there exist methods based on the direct solution of Maxwell's equations ([4]-[7]). These methods make use of numerical techniques such as the Finite Element Method (FEM), the Method of Moments (MoM) or other methods. They are based on fewer neglects than the circuit methods and are thus more accurate. They will therefore be applied for tackling the problem of grounding system modeling.

Third, there exist hybrid methods which tend to combine the circuit theory methods and the electromagnetic methods in order to exhibit the advantages of both ([8]-[9]).

In this paper, the electromagnetic approach is used to calculate fields and transient voltages when lightning strikes a grounding system. The Method of Moments is used to solve the equations of the electromagnetic theory. In the next section, the method is given for a radiating element in a homogeneous dissipative medium.

B. Method of Moments for wire scatterers in a homogeneous lossy medium

The Method of Moments is a general technique for solving integral equations. In the case of electromagnetic problems, we apply the Method of Moments to an integral representation of Maxwell's equations known as Pocklington's equation, written in terms of a scalar potential $\phi$ and a vector potential $\vec{A}$ :

$$
\begin{gathered}
\vec{B}^{s}=\vec{\nabla} \times \vec{A} \\
\vec{E}^{s}=-\vec{\nabla} \phi-j \omega \vec{A} \\
\phi(\vec{r})=\int_{V_{S}} \frac{1}{\varepsilon_{e f f}} \rho_{S}\left(\vec{r}^{\prime}\right) \frac{e^{-\gamma\left\|\vec{r}-\vec{r}^{\prime}\right\|}}{4 \pi\left\|\vec{r}-\vec{r}^{\prime}\right\|} d^{3} \vec{r}^{\prime} \\
\vec{A}(\vec{r})=\int_{V_{S}} \mu \vec{J}_{S}\left(\vec{r}^{\prime}\right) \frac{e^{-\gamma\left\|\vec{r}-\vec{r}^{\prime}\right\|}}{4 \pi\left\|\vec{r}-\vec{r}^{\prime}\right\|^{3}} d^{3} \vec{r}^{\prime}
\end{gathered}
$$

The equations state the relationship between charges and currents on the one hand, and the scattered electric field $\vec{E}^{S}$ and the scattered magnetic induction $\vec{B}^{S}$ on the other hand. The charges have a volume density $\rho_{s}$ in volume $V_{s}$ and the currents have a surface density $\vec{J}_{s}$ in $V_{s}$. The quantity $V_{s}$ is called the 'source' region (subscript s) and it contains only free charges and currents. The quantity $\gamma=\frac{\omega}{c}=j \omega \sqrt{\mu \varepsilon_{e f f}}$ is the propagation constant. It consists of a real part, the attenuation factor, and an imaginary part, the phase factor. In order to write these expression in this concise form, an effective permittivity $\varepsilon_{\text {eff }}$, equal to $\varepsilon_{\text {eff }}=\varepsilon+\frac{\sigma}{j \omega}$, has to be used instead of the permittivity $\varepsilon$. Furthermore, the source region $V_{s}$ has to coincide with the volume of the scatterer.

In its simplest form, the Method of Moments is applied to wire scatterers. Since the grounding systems of interest in this study consist of rods, loops and grids, they can be modeled as wire structures. To be able to numerically solve the equations (1)-(4), the wire structure is divided in smaller segments. A thin-wire assumption is made: it is assumed that the radius of the wire is much smaller (more than ten times smaller) than the length of a segment. The wire scatterers are discretized in $N$ segments. The $n^{\text {th }}$ segment is identified by its starting point, $n^{-}$, its midpoint, $n$ and its termination point $n^{+}$. The interval $\Delta l_{n}$ denotes the length of the line segment terminated by $n$ and $n^{+}$.

It can be shown that the one-dimensional, discretized form of equations (1)-(4) can be written in matrix form ([10], where a good introduction to the Method of Moments is given) as:

$$
[V]=[Z]\left[I_{S}\right]
$$

Where

$$
\left[I_{S}\right]=\left[\begin{array}{c}
I_{S}(1) \\
I_{S}(2) \\
\vdots \\
I_{S}(N)
\end{array}\right] \text { and } \quad[V]=\left[\begin{array}{c}
\vec{E}^{i}(1) \cdot \overrightarrow{\Delta l_{1}} \\
\vec{E}^{i}(2) \cdot \overrightarrow{\Delta l_{2}} \\
\vdots \\
\vec{E}^{i}(N) \cdot \overrightarrow{\Delta l_{N}}
\end{array}\right]
$$

are the vectors with the source currents through each segment and the voltages across each segment. Quantity $\vec{E}^{i}(k)$ represents the electric field incident on segment $k$. The mutual impedance between elements $m$ and $n$ is defined as the voltage across $m$ divided by the cause of this voltage, the current through $n$ :

$$
\begin{aligned}
Z_{m n} \equiv & \frac{\vec{E}^{i}(m) \cdot \overrightarrow{\Delta l_{m}}}{I_{S}(n)} \\
= & j \omega \mu \overline{\Delta l_{n}} \cdot \overline{\Delta l_{m}} \psi(n, m)+ \\
& \frac{1}{j \omega \varepsilon_{\text {eff }}}\left[\psi\left(n^{+}, m^{+}\right)-\psi\left(n^{-}, m^{+}\right)-\psi\left(n^{+}, m^{-}\right)+\psi\left(n^{-}, m^{-}\right)\right]
\end{aligned}
$$

It can be seen that the impedance of segment $m$ due to segment $n$ consists of five terms. The first term is the influence of the magnetic vector potential on the electric field and the last four terms represent the influence of the scalar potential on the electric field. The function $\psi(n, m)$ is given by:

$$
\psi(n, m)=\frac{1}{\Delta l_{n}} \int_{\Delta l_{n}} \frac{e^{-\gamma R}}{4 \pi R} d l
$$

where $\mathrm{R}$ is the distance between a point on segment $n$ and the midpoint of segment $m$. It should numerically be evaluated.

\section{Influence of the Air-Ground Interface}

When the MoM is used for modeling grounding systems, they are represented as scatterers, and previous section assumed they are radiating in a homogeneous medium. 
However, for grounding rods and grids, this is not correct because they are embedded in the soil but are in close vicinity of the air, which has different electromagnetic characteristics.

This problem was solved with a method, known as the modified image technique.

It states that the electric field in an observation point due to a radiating charge- and current-carrying element which is suspended in the same half-space as the observation point, is approximately equal to the electric field in the situation of Fig. 2.a. There, the scatterer is mirrored with respect to the flat boundary between medium 1 and 2 and the charge and current of the image element are equal to the original charge and current, multiplied with a factor $F$. Furthermore, in the situation of Fig. 2.b, there is only one medium, exhibiting the characteristics of medium 1 . This is the modified image theory and it is proven in [5]. There, factor $F$ is also calculated as:

$$
F=\frac{\varepsilon_{\text {eff } 1}-\varepsilon_{\text {eff } 2}}{\varepsilon_{\text {eff } 1}+\varepsilon_{\text {eff } 2}}
$$

It is an approximate technique and is valid if the distance $R$ between source element and observation point, satisfies the relationship $|\gamma R|<<1$.

$$
\begin{aligned}
& \sum_{\varepsilon_{1}, \mu_{1}, \sigma_{1}}^{Q, I} \text {. observation point } \\
& { }_{\varepsilon_{2}, \mu_{2}, \sigma_{2}} / / / / / / / /
\end{aligned}
$$

Fig. 2.a. We want to know the electrical situation in the observation point when a scatterer in medium 1 is suspended above a medium 2

\section{Dielectrically Insulated Wire Structures}

When a dielectric is subject to an externally applied electric field with a time-varying magnitude, the moving, bound charges produce a current, which is called "polarization current". The problem of modeling dielectric insulations is thus reduced to finding these sources, i.e., the polarization charges and current.

In the derivation of an expression for the polarization charges, the geometrical model of Fig. 3 is assumed. The insulating layer has an inner radius $r_{a}$ and an outer radius $r_{b}$. The notation $r_{a}{ }^{+}$denotes the position on the outer boundary of the metal core, just inside the insulating layer. $r_{a}{ }^{-}$denotes the position on the outer boundary of the core, still inside the metal. An analogous notation $\left(r_{b}^{+}\right.$and $\left.r_{b}{ }^{-}\right)$applies for the boundary between medium 1 and 2 .

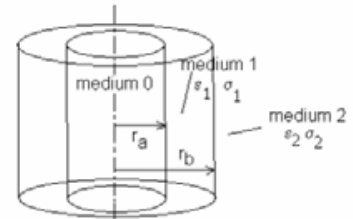

Fig. 3. Geometry of the insulated wire scatterer, modeled by the Method of Moments

The polarization charges only exist on the boundaries $r_{a}$ and $r_{b}$. The surface density of the polarization charges at these two boundaries, is given by, in cylindrical coordinates:

$$
\begin{aligned}
\Sigma_{P}\left(r_{a}\right)=-\hat{r} & .\left(\vec{P}\left(r_{a}^{+}\right)-\vec{P}\left(r_{a}^{-}\right)\right)=-\left(\varepsilon_{1}-\varepsilon_{0}\right) \hat{r} \cdot \vec{E}\left(r_{a}^{+}\right) \\
\Sigma_{P}\left(r_{b}\right) & =-\hat{r} .\left(\vec{P}\left(r_{b}^{+}\right)-\vec{P}\left(r_{b}^{-}\right)\right) \\
& =-\hat{r} .\left(\left(\varepsilon_{2}-\varepsilon_{0}\right) \vec{E}\left(r_{b}^{+}\right)-\left(\varepsilon_{1}-\varepsilon_{0}\right) \vec{E}\left(r_{b}^{-}\right)\right)
\end{aligned}
$$

Quantity $\hat{r}$ is the unit radial vector and $\vec{P}$ is the polarization density vector. Equation (11) can be further simplified by substituting $\vec{E}\left(r_{b}^{+}\right)$by $\vec{D}\left(r_{b}^{+}\right) / \varepsilon_{2}$ and making use of the boundary condition $\vec{D}\left(r_{b}^{+}\right) \cdot \hat{r}=\Sigma_{f}\left(r_{b}\right)+\hat{r} \cdot \vec{D}\left(r_{b}^{-}\right)$. Here, $\Sigma_{f}\left(r_{b}\right)$ is the surface density of the free charges at boundary $r_{b}$. This density of free charges is found by imposing continuity of the radial component of the total current density $\vec{J}_{\text {tot }}=(\sigma+j \omega \varepsilon) \vec{E}$ across the boundary $r_{b}$ :

$$
\hat{r} .\left(\sigma_{1}+j \omega \varepsilon_{1}\right) \vec{E}\left(r_{b}^{-}\right)=\hat{r} .\left(\sigma_{2}+j \omega \varepsilon_{2}\right) \vec{E}\left(r_{b}^{+}\right)
$$

and substituting in this relation $\vec{E}\left(r_{b}^{+}\right)$by $\vec{D}\left(r_{b}^{+}\right) / \varepsilon_{2}$ and again making use of the boundary condition for $\vec{D}$, introducing $\Sigma_{f}\left(r_{b}\right)$ in the expressions. We find:

$$
\begin{gathered}
\Sigma_{f}\left(r_{b}\right)=\varepsilon_{2}\left(\frac{\varepsilon_{1, \text { eff }}}{\varepsilon_{2, \text { eff }}}-\frac{\varepsilon_{1}}{\varepsilon_{2}}\right) \hat{r} \cdot \vec{E}\left(r_{b}^{-}\right) \\
\Sigma_{P}\left(r_{b}\right)=\hat{r} \cdot \vec{E}\left(r_{b}^{-}\right)\left(\left(\varepsilon_{1}-\varepsilon_{0}\right)+\frac{\varepsilon_{1, \text { eff }}}{\varepsilon_{2, \text { eff }}}\left(\varepsilon_{0}-\varepsilon_{2}\right)\right)
\end{gathered}
$$

Expressions (10) and (13) still contain unknown quantities: the radial component of the electric field inside the dielectric layer. This electric field can be calculated with Ampère's law for an integration path inside the insulation:

$$
\oint \vec{H} \cdot d \vec{l}=\iint(\vec{J}+j \omega \vec{D}) \overrightarrow{d S}
$$

Let's take as integration path a circle with radius $r$ $\left(r_{a} \leq r \leq r_{b}\right)$ and centre on the conductor's axis. $\vec{D}$ is radially oriented and therefore vanishes in Ampère's law:

$$
H_{\varphi}=\frac{I\left(z^{\prime}\right)}{2 \pi r}
$$

Ampère's law, written in differential form, is:

$$
\vec{\nabla} \times \vec{H}=\vec{J}+j \omega \vec{D}
$$

For a point inside the dielectric, the electric field is:

$$
\vec{E}(r)=\frac{1}{j \omega \varepsilon_{2}} \hat{r} \frac{\Lambda_{S} \omega}{2 \pi r}, \quad r_{a}<r<r_{b}
$$

$\Lambda_{S}$ is the line density of the source charges. Thus, we find expressions for the free charges and the polarization charges at $r_{b}$ and at $r_{a}$. What is important, is the sum of the free and the bound (polarization) charges at $r_{b}$ and $r_{a}$. The surface densities are:

$$
\begin{gathered}
\Sigma_{f}\left(r_{b}\right)+\Sigma_{P}\left(r_{b}\right)=\frac{\Lambda_{S}}{2 \pi r_{b} \varepsilon_{1}}\left(\varepsilon_{0} \frac{\varepsilon_{1, e f f}-\varepsilon_{2, e f f}}{\varepsilon_{2, e f f}}\right) \\
\Sigma_{f}\left(r_{a}\right)+\Sigma_{P}\left(r_{a}\right)=\frac{\Lambda_{s} \varepsilon_{0}}{2 \pi r_{a} \varepsilon_{1}}
\end{gathered}
$$


With these surface charge densities, the scalar potential can be calculated:

$$
\phi(\vec{r})=\frac{1}{4 \pi \varepsilon_{2, \text { eff }}} \int_{a x i s} \Lambda_{S}\left(z^{\prime}\right)\left(\int_{\varphi=0}^{2 \pi}\left(C_{a} \frac{e^{-\gamma R_{a}}}{R_{a}}+C_{b} \frac{e^{-\gamma R_{b}}}{R_{b}}\right) \cdot d \varphi\right) d z^{\prime}
$$

where $C_{a}$ en $C_{b}$ are constants, only depending on $\varepsilon_{1, \text { eff }}$,

$\varepsilon_{2, \text { eff }}$ and $\varepsilon_{1}$. This equation can be used instead of (3) and

the impedance terms $Z_{m n}$ of the MoM-equation (5) can again be calculated. The surface integral of (20) is numerically evaluated by placing point charges every $\Delta \theta=10$ degrees around the surface of the cylinder.

\section{Application of the Moment Method to the Problem} OF ANALYZING LigHTNING STRIKES ON GROUNDING SYSTEMS

\section{A. General considerations}

The method of moments can be used to analyze the effectiveness of grounding systems when a lightning strikes the system. Consider a grounding system which is divided into $N$ small segments. A lightning that strikes the system can be modeled as a current source which injects the lightning current. The source is placed in the $k^{\text {th }}$ segment.

When impedances are present in the grounding system, other than the impedances of the grounding system itself, e.g. current or surge arresters or isolation transformers, they can be included in the model by adding them to the corresponding diagonal element of the impedance matrix $Z$.

Because the grounding system is excited with a current source on the $k^{\text {th }}$ segment, we can write following matrix equation:

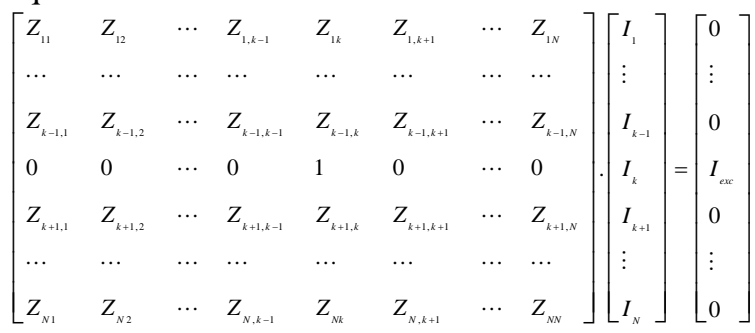

This matrix equation states that the voltage across every segment is equal to zero, except for the $k^{\text {th }}$ row. This row expresses that the current on segment $k$ equals $I_{e x c}$, which is the amplitude of a sinusoidal excitation current on the $k^{\text {th }}$ segment. $I_{i}, i=1, \ldots, N$, is the amplitude of the sinusoidal current of the $i^{\text {th }}$ segment. The right-hand side of equation (21) is the vector with known quantities. The elements of the impedance matrix are also known. This linear system has to be solved in order to obtain the vector with the unknown currents.

When the current on each segment is known, the scalar potential in an arbitrary point inside the ground can be calculated. This scalar potential can be used as the definition of the voltage between the point $\vec{r}$ and the far earth. The voltage between the points 1 and 2 is defined as:

$$
V_{21}=V_{2}-V_{1}=\phi_{2}-\phi_{1}
$$

The Method of Moments works in the frequency domain. This means that the input and output data must be pre- and postprocessed in order to calculate the transient behavior of e.g. the voltage in a point on a grounding electrode. The Fourier transform is applied to obtain the frequency components of a lightning current. For each frequency, the current vector of (21) and the potential is to be computed. In (21), the Fourier component of the lightning current at that frequency is to be used. After all the frequency components of the voltage in a point are collected, the inverse Fourier transform is used to transform the signal to the time domain. This gives the transient voltage.

The transient impedance of a grounding system can then be calculated by dividing the transient voltage at the current injection point by the lightning current. Another important quantity which can be computed with the described MoMbased technique, is the step voltage on the earth's surface. This step voltage is equal to the electric field $(\mathrm{V} / \mathrm{m})$ on the earth's surface above the grounding system, and can be calculated with (2), after the source charges and currents are known.

\section{B. Information about the Applied Lightning Currents}

The lightning current is represented as a double-exponential wave:

$$
i(t)=k I_{\max }\left(e^{-\alpha t}-e^{-\beta t}\right)
$$

According to the German standard VDE V 0185-1 ([13]), positive lightning strikes occur in $10 \%$ and negative lightning in $90 \%$ of all cases. A representative positive surge is the 200 $\mathrm{kA}$ wave with a rise time of $10 \mu$ s and a time-to-half of 350 $\mu$ s. It has parameters $k, I_{\max }, \alpha$ and $\beta$ as specified in Table I in the column 'wave 1'. Also the parameters of a representative negative main surge ('wave 2') and a subsequent negative surge ('wave 3') are given in this table.

TABLE I

PARAMETERS OF LIGHTNING CURRENT WAVES ACCORDING TO VDE V 0185-1

\begin{tabular}{|l|l|l|l|}
\hline & Wave 1 & Wave 2 & Wave 3 \\
\hline rise time $[\mu \mathrm{s}]$ & 10 & 1.5 & 0.25 \\
\hline time-to-half $[\mu \mathrm{s}]$ & 350 & 150 & 100 \\
\hline$k$ & 1.0514 & 1.0225 & 1.0054 \\
\hline$I_{\max }[\mathrm{kA}]$ & 200 & 150 & 50 \\
\hline$\alpha\left[(\mu \mathrm{s})^{-1}\right]$ & 0.0021271 & 0.0057275 & 0.0069865 \\
\hline$\beta\left[(\mu \mathrm{s})^{-1}\right]$ & 0.24606 & 1.7338 & 10.8228 \\
\hline$f_{99.9}[\mathrm{~Hz}]$ & 42178 & 192072 & 460392 \\
\hline
\end{tabular}

An interesting parameter is $f_{99.9}$. This is the frequency so that $99.9 \%$ of all energy of the double-exponential lightning current is in its spectrum between 0 en $f_{99.9} \mathrm{~Hz}$. It is used as definition for the maximum frequency in the current signal. Sampling is done at a rate of $2.5 f_{99.9}$.

\section{Application OF the Moment for ANALYSing INFRABEL’S GROUNDING SYSTEM}

\section{A. Description of the simulation model}

For the validation of the method, we refer to [14]. The grounding topologies and lightning currents of [8], [7], and [6] were simulated with the method of this work and results 
were in good comparison the measured or simulated results of [8], [7], and [6]. Also, some situations for which there exist analytical expressions, were simulated in [14].

The problem, presented in Fig. 1, is modeled by the wireframe shown in following figures. In Fig. 4, the segmented grounding system of the BTS is shown. It consists of a copper grounding loop with four vertical electrodes (diameter 20 $\mathrm{mm})$. There is possibility to insert an isolation transformer in the model. The surge arresters (Dehnguard and Dehnbloc) are also included. They are modeled as constant resistors of $0.1942 \Omega$ and $0.2168 \Omega$ respectively. There are three points where the lightning current is injected. It is assumed that the current is evenly distributed over these three points. These points correspond with the three sides of the pyramidal GSMtower, which is not modeled. Point R1 is also indicated (cfr. Fig. 1). EXVB is in Belgium a type of cable with a core of 16 $\mathrm{mm}^{2}$ and around it an insulation of cross-linked polyethylene (XLPE) of $0.7 \mathrm{~mm}$. H07V-R is a cable with core of $50 \mathrm{~mm}^{2}$ with around it an PVC insulation of thickness $1.4 \mathrm{~mm}$.

The EXVB cable is the connection between the BTS and the supply post. The grounding system of the supply station is shown in Fig. 5. There is also a possibility to include the frequency-dependent impedances of an isolation transformer. The point Rs is also present (cfr. Fig. 1). The supply station has a single vertical grounding rod, connected to the cable with a current surge arrestor.

\section{B. Simulation results}

In this section, the influence of electrical and grounding topology parameters on the protection against lightning is examined. Graphs show the results of the parametric sweeps. If the parameter is not swept, then the simulations are done with

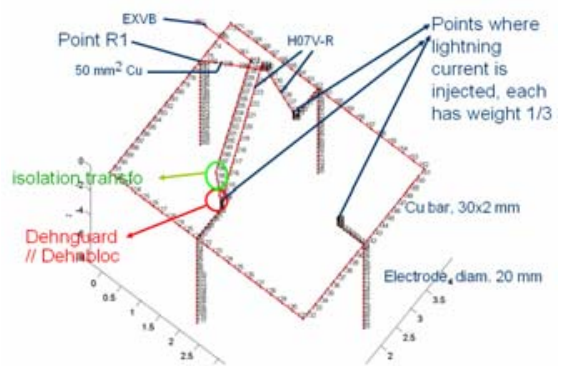

Fig. 4. Wireframe model of the Base Transceive Station’s grounding system.

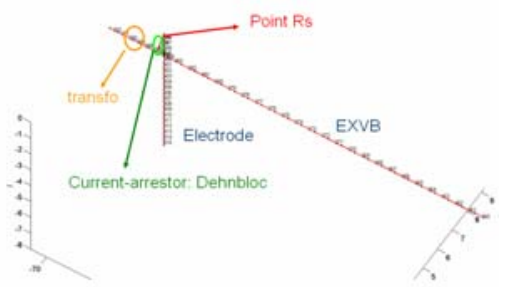

Fig. 5. Wireframe model of the supply station's grounding system.

default values. The default value of the length of the EXVB cable is $70 \mathrm{~m}$. The default length of the grounding rods is $7 \mathrm{~m}$. The default soil resistivity is $100 \Omega \mathrm{m}$ and its default permittivity is $10 \varepsilon_{0}$. All sweeps are done with the three types of lightning current (Table I).
In Fig. 6a, the transient voltage at point Rs is shown for the $10 / 350$ us-lightning current (200 kA) when the length of the EXVB cable is varied. Fig. 6b. shows the same sweep, but now the voltage at R1 is calculated. In Fig. 7 the voltage at Rs is shown for different values of the soil resistivity. Fig 8a and Fig. 8b show the voltage at Rs, when the length of the grounding rods of the BTS, respectively of the supply station, varies. Finally, Fig. 9a and Fig. 9b show the voltage at R2 and $\mathrm{R} 1$ when there is an extra grounding rod halfway between Rs and R1. It is called R2 and is connected with R1 with a bare cable, or with the EXVB cable through a surge arrestor.

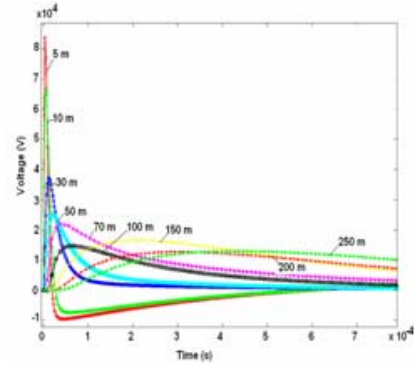

Fig. 6.a. Voltage at Rs for varying EXVB cable length and 10/350 $\mu \mathrm{s}$ lightning. EXVB cable length and 10/350 $\mu \mathrm{s}$ lightning.

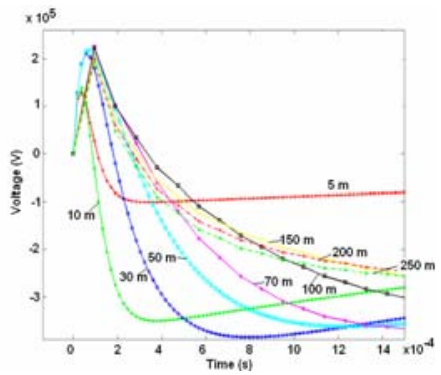

Fig.6.b. Voltage at R1 for varying

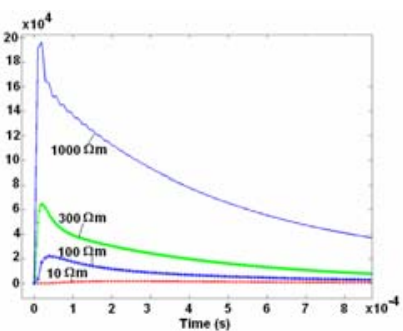

Fig. 7. Voltage at Rs for varying soil resistivity and 10/350 $\mu$ s lightning.

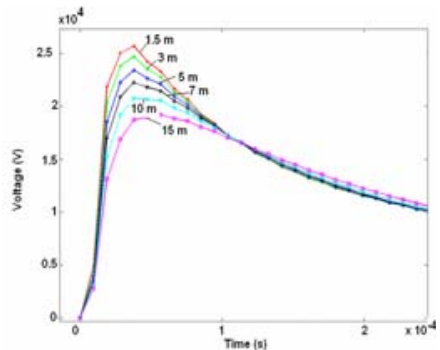

Fig. 8.a. Voltage at Rs for varying length of BTS-electrodes and 10/350 $\mu$ s lightning

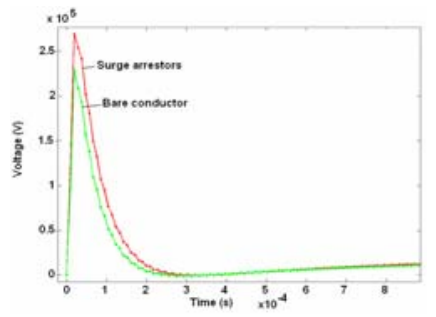

Fig. 9.a. Voltage at R2 when grounding electrode at R2 is present and for a $10 / 350 \mu$ s lightning.

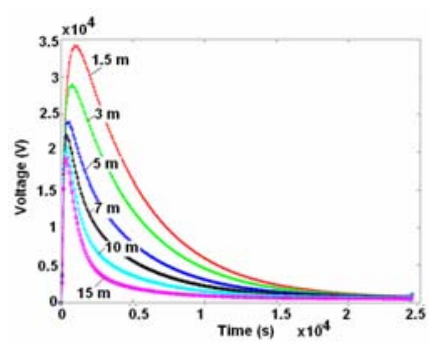

Fig. 8.b. Voltage at Rs for varying length of electrodes of the supply station and 10/350 $\mu$ s lightning

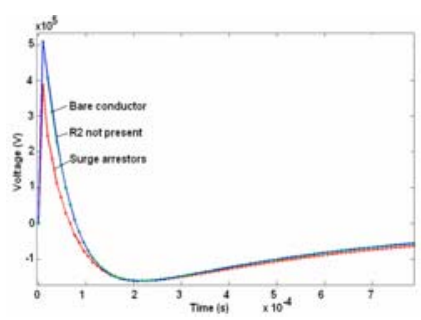

Fig. 9.b. Voltage at R1 when grounding electrode at R2 is or isn't present and for a $10 / 350 \mu$ s lightning
From the simulation results, it can be seen that the voltage at R1 suffers from transmission line effects because of the very branched structure of the BTS grounding system. The 
reflections on points where the impedance changes lead to very high voltages $(\sim 100 \mathrm{kV})$ at R1. If an isolation transformer is used, it is best placed in the supply station.

For short cable lengths $(<30 \mathrm{~m})$, the transmission line effect is clearly visible. The voltages at Rs are very high $(\sim 70-80$ $\mathrm{kV}$ ) en change polarity. When the length of the EXVB cable is $100 \mathrm{~m}$ or more, the decrease of the peak voltage at Rs is not so important anymore: the difference between $100 \mathrm{~m}$ and 250 $\mathrm{m}$ is small.

Although in this paper the simulations with the $10 / 350 \mu \mathrm{s}$ pulse were shown, it is seen that for current pulses with a short rise time, the effect of this short rise time is mainly visible for short cable lengths $(<30 \mathrm{~m})$. The peak voltage increases highly in comparison with pulses with a longer rise time. Because these steep current pulses have a smaller amplitude, a cable longer than $30 \mathrm{~m}$ implies a decrease of the peak voltage in comparison with the $200 \mathrm{kA}$ pulse.

The soil resistivity has an important influence. There is a huge difference between 10, 100 and $300 \Omega \mathrm{m}$.

When the length of the BTS-electrodes varies between 1.5 and $15 \mathrm{~m}$, the voltage at Rs has a variation of $25 \%$. This is not important. However, when the length of the grounding rods of the supply station is varied, the voltage at Rs varies with $50 \%$. This is quite an important change, and it is therefore worth increasing the grounding rod's length.

Whether or not the intermediate grounding R2 is present, does not affect the voltage at Rs or R1. However, if R2 is present, the lowest potential at R2 can be obtained when R2 is directly connected with R1 with a bare copper connection.

\section{CONCLUSION}

In this work, a method was presented to simulate the behavior of grounding systems struck by a lightning current. It is based on numerically solving Maxwell's equations with the Method of Moments. First, the method was presented for wire scatterers in a single space. Then, the method is extended to model the presence of the air-ground interface layer. Next, a second extension is made in order to be able to simulate dielectrically insulated wires. The method was applied on a problem which Infrabel, the Belgian railway infrastructure manager, has for the grounding of GSM-R base stations.

\section{REFERENCES}

[1] M. I. Lorentzou and N. D. Hatziargyriou, "Transmission line modeling of grounding electrodes and calculation of their effective length under impulse excitation," presented at the International Conference on Power Systems Transients (IPST’05), Montreal, Canada, 2005.

[2] F. E. Menter and L. Grcev, "EMTP-based model for grounding system analysis," IEEE Transactions on Power Delivery, Vol. 9, No. 4, pp. 1838-1849, October 1994.

[3] J. Van Waes, "Safety and EMC aspects of grounding - experimental studies in high-power systems," Ph.D. dissertation, Technische Universiteit Eindhoven, 2003.

[4] L. Grcev and F. Dawalibi, "An electromagnetic model for transients in grounding systems,” IEEE Transactions on Power Delivery, Vol. 5, No. 4, pp. 1773-1781, Nov. 1990.

[5] G. Ala and M. L. Di Silvestre, "A simulation model for electromagnetic transients in lightning protection systems," IEEE Transactions on Electromagnetic Compatibility, Vol. 44, No. 4, pp. 539-554 ,Nov. 2002.

[6] L. D. Grcev and M. Heimbach, "Frequency dependent and transient characteristics of substation grounding system," IEEE Transactions on Power Delivery, Vol. 12, pp. 172-178, Jan. 1997
[7] L. Grcev, "Computer analysis of transient voltages in large grounding systems," IEEE Transactions on Power Delivery, Vol. 11, pp. 815-823, April 1996.

[8] Y. Liu, M. Zitnik and R. Thottappillil, "An improved transmission-line model of grounding system," IEEE Transactions on Electromagnetic Compatibility, Vol. 43, No. 3, pp 348-355, August 2001.

[9] M. Heimbach and L. D. Grcev, "Grounding system analysis in transients programs applying electromagnetic field approach,” IEEE Transactions on Power Delivery, Vol. 12, NO. 1, pp. 186-193, Jan. 1997.

[10] R. F. Harrington, Field computation by moment methods, New York: MacMillan, 1968, ch. 4

[11] A. Sommerfeld, Vorlesungen über Theoretische Physik VI. Partielle Differentialgleichungen in der Physik, Wiesbaden: Auflage Diederich, 1947.

[12] P. E. Atlamazoglou and N. K. Uzunoglu, "A Galerkin moment method for the analysis of an insulated antenna in a dissipative dielectric medium,” IEEE Transactions on Microwave Theory and Techniques, Vol. 46, No. 7, pp. 988-996, July 1998.

[13] Blitzschutz - Teil 1: Allgemeine Grundsätze, VDE Standard Teil $12002-$ 11 DIN V VDE V 0185-1, 2002

[14] P. Jacqmaer and J. Driesen, "Modelling of grounding systems with the method of moments," in Proc. of 3rd IEEE Benelux Young Researchers Symposium in Electrical Power Engineering, Gent, Belgium, April 2728, 2006, 10 pages.

\section{BIOGRAPHIES}

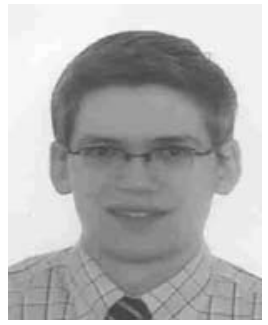

Pieter Jacqmaer was born in 1980 in Belgium. He received the M Sc degree in Electronics Engineering in 2003 and the M Sc degree in Electrotechnical Engineering in 2007 from the Katholieke Universiteit Leuven (K.U.Leuven), Belgium and is currently pursuing a $\mathrm{Ph} \mathrm{D}$ in Electrotechnical Engineering at the Research Group ELECTA, K.U.Leuven. His main interests are power electronics, control strategies for circuits and drives and electromagnetic compatibility. His research focuses on the development of circuits for high-frequency switching devices such as new wide-bandgap power electronic components, allowing them to operate under lower electrical tensions and with less electromagnetic interference.

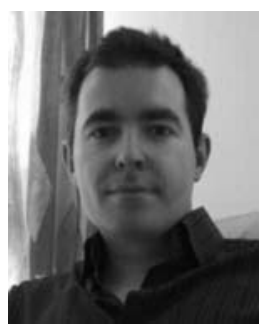

Christophe Geuzaine received received his MSc degree in 1996 and his PhD degree in 2001, both from the Faculty of Applied Sciences at the University of Liège in Belgium. After post-doctoral positions at the California Institute of Technology and with the Belgian National Science Foundation, he became in 2005 an assistant professor of Mathematics at Case Western Reserve University. In 2007 he came back to Belgium and joined the Engineering Faculty at the University of Liège. Prof. Geuzaine's research encompasses modeling, analysis, algorithm development, and simulation for problems arising in various areas of engineering and science, with current applications in low- and high-frequency computational electromagnetics.

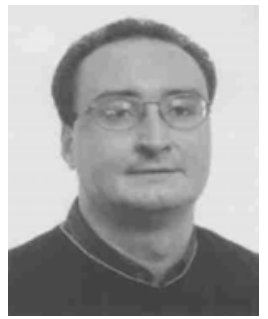

Johan Driesen (S'93, M'97) was born in 1973 in Belgium. He received the M Sc degree in 1996 as Electrotechnical Engineer from the K.U.Leuven, Belgium. He received the $\mathrm{Ph} \mathrm{D}$ degree in Electrical Engineering at K.U.Leuven in 2000 on the finite element solution of coupled thermal-electromagnetic problems and related applications in electrical machines and drives, microsystems and power quality issues. Currently he is an associate professor at the K.U.Leuven and teaches power electronics and drives. In 2000-2001 he was a visiting researcher in the Imperial College of Science, Technology and Medicine, London, UK. In 2002 he was working at the University of California, Berkeley, USA. Currently he conducts research on distributed generation, including renewable energy systems, power electronics and its applications, for instance in drives and power quality. 